\title{
UV OBSERVATIONS OF THREE CLASSICAL NOVAE DURING EARLY STAGES OF DECLINE*
}

\author{
H. DRECHSEL, W. WARGAU and J. RAHE
}

Dr Remeis Sternwarte Bamberg, Astronomisches Institut Universität Erlangen Nürnberg, F.R.G.

\author{
(Received 23 August, 1983)
}

\begin{abstract}
We present IUE UV spectra of the three recent classical novae N Sgr 1982, N Ser 1983, and N Mus 1983, obtained during early decline, transitional stage, and nebular phase, respectively. The line spectra are discussed. Preliminary conclusions concerning CNO over-abundances, shell geometry, and speed classes are drawn.
\end{abstract}

\section{Introduction}

IUE observations of the three recent novae N Sagittarii 1982, N Serpentis 1983, and N Muscae 1983 have been collected between October, 1982 and March, 1983.

N Sgr 1982 was discovered by Honda (1982) on 4 October, 1982 at a visual brightness of 9.0 .0 . The absolute visual maximum of 8.0 was reported for 14 October, 1982. Subsequently, the brightness decreased steeply to $10^{m} \cdot 6$ on 25 October, 1982, and afterwards increased again to $8^{m .5}$ on 9 November, 1982.

The outburst of N Ser 1983 has been reported by Wakuda (1983) on 21 February, 1983, at a visual brightness of $11^{\mathrm{m}} \cdot 0$. Soon after discovery, a peak brightness of $7^{m .7}$ was reached on 22 February, 1983, followed by a very steep decline of about 4 mag within only 6 days.

The outburst of N Mus 1983 was discovered by Liller (1983), at a visual brightness of $7^{m} .2$ on 18 January, 1983. After the brightness maximum of about $7^{m}$ on 21 January, the object declined by about 2 mag within a few days. Subsequent fluctuations around $9^{m}$ were reported until 22 February. Later, the nova declined only gradually, and still was at a visual brightness of $10^{\text {m. }}$ at the beginning of 1983 August (Cassatella, 1983).

Except for $\mathrm{N}$ Mus 1983, our measurements represent the first UV observations of these objects. The satellite observations were carried out between 3 and 44 days after the visual brightness maxima. Since the three classical novae belong to different speed classes, the observing epochs coincide with different stages of the nova events.

\section{IUE Data}

The journal of UV observations of the three novae N Sgr 1982, N Ser 1983, and $\mathrm{N}$ Mus 1983 is presented in Table I, containing the dates of observations, image

\footnotetext{
* Paper presented at the Lembang-Bamberg IAU Colloquium No. 80 on 'Double Stars: Physical Properties and Generic Relations', held at Bandung, Indonesia, 3-7 June, 1983.
} 
TABLE I

Journal of UV Observations

\begin{tabular}{|c|c|c|c|c|c|c|}
\hline Object & Date & (UT) & Camera & $\begin{array}{l}\text { Image } \\
\text { no. }\end{array}$ & Disp. & $\begin{array}{l}\text { Exp. } \\
\text { time } \\
\text { (min.) }\end{array}$ \\
\hline \multirow[t]{13}{*}{ N Sgr 1982} & \multirow[t]{5}{*}{ 1982, Oct. } & 18.637 & SWP & 18323 & low & 5 \\
\hline & & 18.644 & LWR & 14433 & low & 5 \\
\hline & & 18.665 & SWP & 18324 & low & 60 \\
\hline & & 18.710 & LWR & 14434 & low & 20 \\
\hline & & 18.729 & SWP & 18325 & low & 30 \\
\hline & \multirow[t]{4}{*}{1982 , Oct. } & 19.632 & SWP & 18329 & low & 40 \\
\hline & & 19.665 & LWR & 14441 & low & 25 \\
\hline & & 19.684 & SWP & 18330 & low & 30 \\
\hline & & 19.707 & LWR & 14442 & low & 6 \\
\hline & \multirow{4}{*}{ 1982, Oct. } & 31.631 & SWP & 18439 & low & 30 \\
\hline & & 31.655 & LWR & 14530 & low & 30 \\
\hline & & 31.678 & SWP & 18440 & low & 60 \\
\hline & & 31.703 & LWR & 14531 & low & 5 \\
\hline \multirow[t]{10}{*}{ N Mus 1983} & \multirow[t]{10}{*}{ 1983, March } & 4.176 & SWP & 19383 & low & 20 \\
\hline & & 4.192 & LWR & 15421 & low & 5 \\
\hline & & 4.211 & SWP & 19384 & low & 5 \\
\hline & & 4.232 & SWP & 19385 & low & 2 \\
\hline & & 4.250 & SWP & 19386 & high & 120 \\
\hline & & 4.356 & LWR & 15422 & low & 0.5 \\
\hline & & 4.358 & SWP & 19387 & high & 20 \\
\hline & & 4.397 & SWP & 19388 & low & 0.5 \\
\hline & & 4.406 & LWR & 15423 & high & 45 \\
\hline & & 4.441 & SWP & 19389 & high & 12 \\
\hline \multirow[t]{3}{*}{ N Ser 1983} & \multirow[t]{3}{*}{ 1983, March } & 5.252 & LWR & 15427 & low & 40 \\
\hline & & 5.283 & SWP & 19394 & low & 150 \\
\hline & & 5.412 & SWP & 19395 & low & 54 \\
\hline
\end{tabular}

numbers, dispersion modes, and exposure times. All spectra were taken through the large aperture. A total of 13, 10, and 3 spectra were sampled of N Sgr 1982, N Mus 1983, and N Ser 1983, respectively, at the ESA-Vilspa ground station. For $\mathrm{N}$ Mus 1983, 4 high dispersion spectra were obtained, while the other exposures are all in low dispersion.

\section{Results}

\subsection{NOVA SAGITTARII 1982}

IUE low dispersion spectra were taken 3,4 , and 16 days after the visual brightness maximum. The early spectra of 18 and 19 October, 1982, were obtained only very shortly after the maximum, while the observations of 31 October were made in an ascending part of the light curve following an intermediate brightness minimum. The spectra taken on two consecutive days are very similar, and reveal only minor 


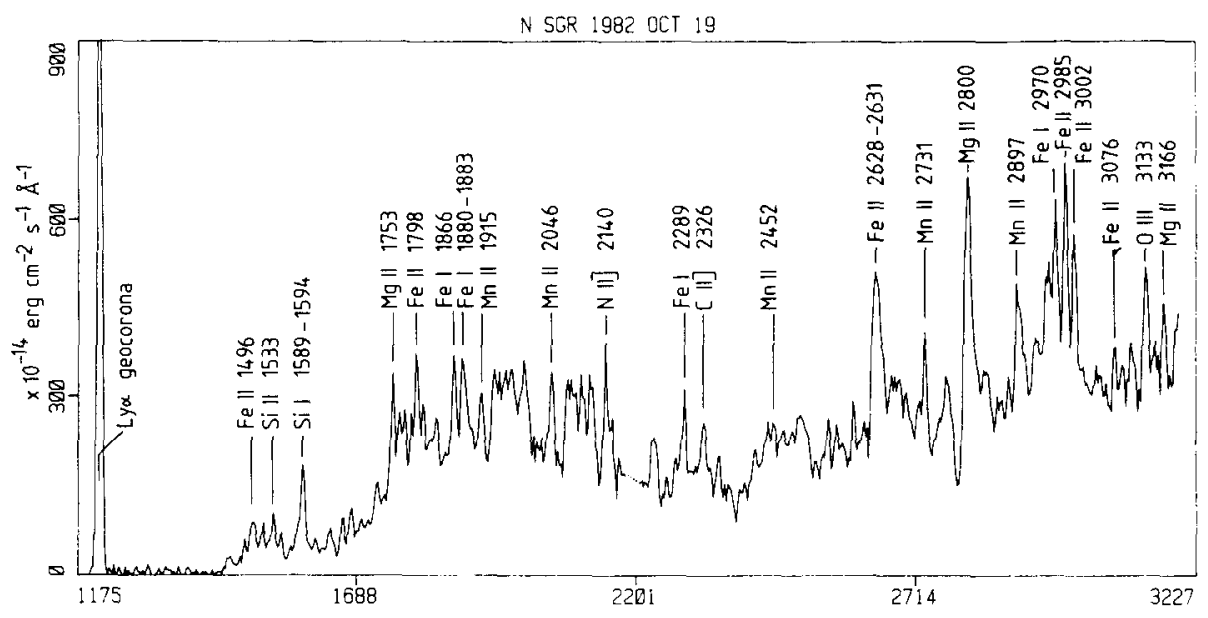

N SER 1983 MARCH 5
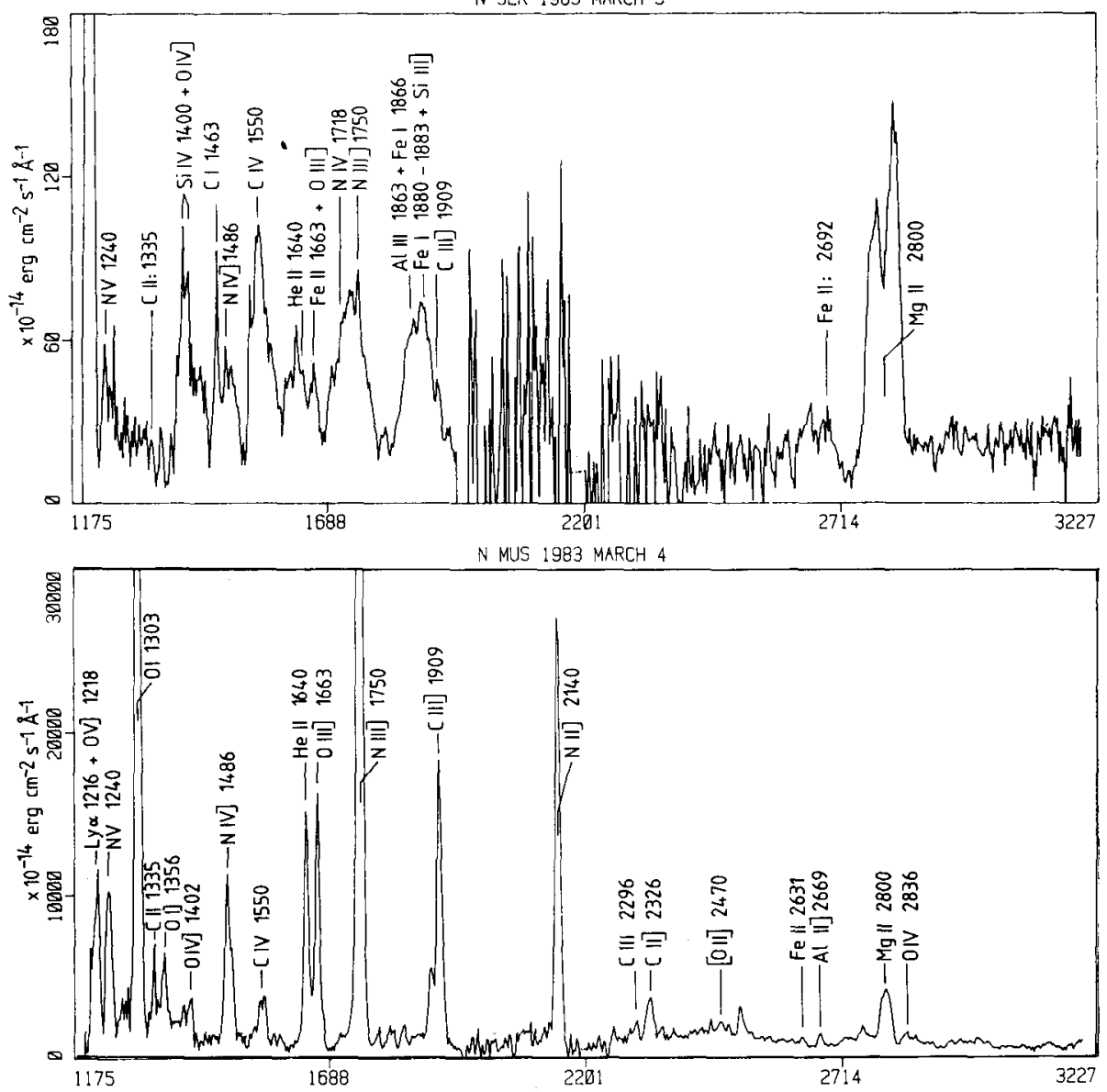

Fig. 1. IUE UV spectra of the three classical novae N Sgr 1982, N Ser 1983, and N Mus 1983 (from top to bottom); the spectra are absolutely calibrated, and have been de-reddened using $E(B-V)=0^{m} \cdot 30,0^{m} \cdot 40$, and $0^{m} .50\left( \pm 0^{m} \cdot 1\right)$, respectively. 
changes in the appearance of the line spectrum, whereas modifications of the relative line intensities and of the continuum intensity distribution are apparent at the later nova stage on 31 October (cf. Drechsel et al., 1982; Blades and Drechsel, 1982).

The absolutely calibrated combined SWP and LWR spectra of N Sgr 1982 of 18 October, 1982, covering the wavelength range between $117.5 \mathrm{~nm}$ and $320 \mathrm{~nm}$, are shown in the upper part of Figure 1. Correction for interstellar extinction has been made using a value of $E(B-V)=0^{m} .30\left( \pm 0^{m} .1\right)$, and applying the extinction curve of Seaton (1979).

The continuum flux decreases steeply between $170 \mathrm{~nm}$ and $160 \mathrm{~nm}$ toward zerolevel at shorter wavelengths, while it increases toward the optical range, as is usually observed for novae in an early phase after outburst maximum; earlier UV observations of the classical novae FH Ser 1970 (Andersen et al., 1971), and N Cyg 1978 (Stickland et al., 1979) showed that the maximum of intensity distribution gradually shifts toward shorter wavelengths in the UV with progressing evolutionary stage of the nova.

The hump between about $170 \mathrm{~nm}$ and $220 \mathrm{~nm}$ is presumably due to the blending of numerous strong emission lines. The spectrum is dominated by permitted emission lines of neutral or singly-ionized metals (e.g., Fe I, Fe II. Si I, Si II. Mg II, $\mathrm{Mn}$ Il, etc.), as is typical for novae in early stages after outburst. The resonance transition of $\mathrm{Mg}$ II at $280 \mathrm{~nm}$ appears as a distinct $\mathrm{P}$ Cygni line, with a terminal velocity of about $-1700 \mathrm{~km} \mathrm{~s}^{-1}$, indicating the existence of an expanding envelope.

The general appearance of the UV spectrum has not drastically changed until 31 October 1982, when the continuum already extends further into the far UV, and the intensities of most emission lines have decreased, except for the semi-forbidden lines of $\left.\mathrm{N}_{\mathrm{II}}\right](214.0 \mathrm{~nm})$ and $\left.\mathrm{C}_{\mathrm{II}}\right](232.6 \mathrm{~nm})$, which strengthen with time.

\subsection{NoVA SERPENTIS 1983}

The IUE spectra of $N$ Ser 1983 were taken 11 days after the sharp brightness maximum, when the object had already declined by more than 4 mag. The visual observations show that N Ser 1983 was obviously a very fast nova, with a $t_{3}$-time of only about 5 days.

The combined absolutely calibrated SWP and LWR spectra, taken on 5 March, 1983, are shown in the middle part of Figure 1. The data have been de-reddened using a value of $E(B-V)=0^{m} 4\left( \pm 0^{m} \cdot 1\right)$. The continuum flux in the UV rises towards the optical range. Due to the poor signal/noise ratio, neither continuum nor emission lines can be detected between about $200 \mathrm{~nm}$ and $240 \mathrm{~nm}$. A significant flux level in the short wavelength (SWP) range could in principle partly be due to some continuum radiation, but is almost certainly produced by the blending of numerous emission lines. A wealth of strong and broad emission lines is present: resonance lines of moderate to high ionization levels (e.g., of $\mathrm{Al} \mathrm{III.}$ $\mathrm{C}$ IV, Si Iv, and $\mathrm{N} \mathrm{v}$ ); semi-forbidden intercombination lines (e.g., of $\mathrm{C} \mathrm{III}$, $\mathrm{N}_{\mathrm{III}}$ ], or 
$\mathrm{O}$ III]); as well as permitted lines of neutral and singly-ionized metals such as $\mathrm{Fe} \mathrm{I}$, $\mathrm{Fe}_{\mathrm{II}}$, or $\mathrm{C}_{1}$ : the recombination line of $\mathrm{He}_{\mathrm{II}}$ at $164 \mathrm{~nm}$ is weak. The most prominent emission feature in the long wavelength (LWR) range is due to the resonance doublet of $\mathrm{Mg}$ II at $280 \mathrm{~nm}$. It is composed of two distinct (blue- and red-shifted) components with different strengths and peak radial velocities of -1520 and $+2250 \mathrm{~km} \mathrm{~s}^{-1}$, respectively, which indicate the expansion of a non-spherically symmetric shell expelled by the nova. The amazingly intense $\mathrm{Mg}$ I-line shows a distinct $\mathrm{P}$ Cygnitype profile, with a terminal velocity - corresponding to the shortward absorption edge - as high as about $-11000 \mathrm{~km} \mathrm{~s}^{-1}$. The $C_{\text {Iv }}(155 \mathrm{~nm})$ line also exhibits a P Cygni profile with a terminal velocity of about $-9000 \mathrm{~km} \mathrm{~s}^{-1}$. The general appearance of the short wavelength IUE spectrum resembles that of Nova Aquilae 1982 (Snijders et al., 1982).

The line spectrum is composed of lines with a wide range in ionization potential, and consists of permitted lines, which are typical for the early decline stage, as well as of semi-forbidden lines usually observed during the nebular phase. Our UV observations were therefore probably made in the transitional stage of the nova.

\subsection{Nova MUSCAE 1983}

The high and low dispersion spectra of $\mathrm{N}$ Mus 1983 were obtained 44 days after the visual brightness maximum, when the nova had declined by about 2.5 mag. The visual observations show that $\mathrm{N}$ Mus 1983 is probably a slow nova. After a relatively steep decline by about 2 mag within a few days, the object remained about constant in brightness at about $9^{m}$, for more than 30 days. At the time of our observations, its brightness was $9^{m} \cdot 6$.

The combined low dispersion SWP and LWR spectra, taken on 4 March, 1983, are shown in the lower part of Figure 1. The data have been de-reddened according to $E(B-V)=0^{m} \cdot 5\left( \pm 0^{m} \cdot 1\right)$. Almost no continuum flux is detectable. The spectrum is dominated by semi-forbidden emission lines of $\mathrm{C}_{\text {III }}$, $\left.\left.\mathrm{C}_{\text {III }}\right], \mathrm{N}_{\text {II }}\right], \mathrm{N}_{\text {III }}$ ], $\mathrm{N}_{\text {IV }}$ ], and $\mathrm{O}$ III]. Lines with relatively high ionization level such as $\mathrm{He}$ II, $\mathrm{C}$ IV, $\mathrm{Nv}, \mathrm{O}$ IV], and $\mathrm{Ov}$ ] are also present. The large number and relative strength of the intercombination lines suggest that the nova had already entered its nebular stage. Comparison can be made with Nova Cygni 1978: the spectral evolution of this nova was followed with IUE from very early phases into the nebular stage, where the appearance of its line spectrum was strikingly similar to that of N Mus 1983 (see, e.g., Figure la of Stickland et al., 1981).

The relative strength, especially of the nitrogen lines, but also of those of carbon and oxygen, suggests very high abundances of these elements, as can be achieved through the operation of the CNO cycle, and in accordance with theoretical models of the nova outburst. A detailed analysis of the high dispersion spectra will yield quantitative abundance results. The overabundance of $\mathrm{C}, \mathrm{N}$, and $\mathrm{O}$ with respect to solar values is estimated to be at least of 1 or 2 orders of magnitude. 


\section{Discussion}

The UV spectra of the three novae N Sgr 1982, N Ser 1983, and N Mus 1983 are compared in Figure 1. The spectral appearance together with the photometric evolution allows a classification of the objects according to their speed class.

The spectrum of N Sgr 1982 shown in the upper part of the figure is typical for early stages after the (visual) outburst maximum: a wealth of neutral and singlyionized metal lines are seen; the continuum decreases toward shorter wavelengths, and drops to zero already in the mid-UV; while the UV spectra of 1982, 18 and 19 October (about 14 days after maximum) are very similar, the one of 31 October shows already a definite shift of the maximum of intensity distribution toward far UV wavelengths. Comparison with the UV spectroscopic evolution of the fast Nova Cygni 1978 (Stickland et al., 1979) suggests that N Sgr 1982 belongs to the class of classical fast novae, and was observed during its early decline prior to the nebular phase.

The spectrum of $N$ Ser 1983 in the middle part of Figure 1 was probably taken during the transitional stage. Lines of low and high-ionization levels as well as resonance, permitted and semi-forbidden lines do coexist. Since this spectrum was taken only 11 days after the sharp visual brightness maximum, N Ser 1983 is probably a very fast nova; this can also be anticipated from the exceptionally short $t_{3}$-time of less than 5 days.

The P Cygni profile of $\mathrm{Mg}$ II $(280 \mathrm{~nm})$ indicates a very strong and fast wind $\left(\sim 10000 \mathrm{~km} \mathrm{~s}^{-1}\right)$ with asymmetric structure. Two blobs or clouds of material contained in the expanding shell can possibly cause the two emission components with a peak separation of several thousand $\mathrm{km} \mathrm{s}^{-1}$. The existence of $\mathrm{Mg}$ II together with high ionization species such as $\mathrm{C}$ IV, $\mathrm{N}$ IV, or $\mathrm{Si}$ IV implies that these ions are formed in spatially separated parts of the wind, which is optically thin and has a large temperature gradient. The high-ionization lines have no doublepeaked emission comparable to that of $\mathrm{Mg}$ ir. They are conceivably formed in deeper layers of the expelled envelope, which do not reflect the asymmetric structure of the outer shell.

The UV spectrum of N Mus 1983 in the lower part of Figure 1 was obtained 44 days after visual maximum light. The spectral appearance is typical for the nebular phase, as one can readily derive from the line spectrum and from a comparison with the spectral evolution of N Cyg 1978 in the UV range (Stickland et al., 1979, 1981). Though the relatively early onset of the nebular phase would lend support to the classification of N Mus 1983 as a moderately fast nova, this is in contradiction with the phtometric development: in early August 1983, some 200 days after maximum, the visual brightness was still at $10^{\mathrm{m}} .9$, which is less than 4 mag below the maximum brightness, and corresponds to a decline rate typical for a slow nova.

Another striking feature of $\mathrm{N}$ Mus 1983 is the exceptional strength of the nitrogen lines, and - to somewhat less extent - also of the carbon and oxygen 
lines. The enhancement of nitrogen relative to the Sun, amounts to more than two orders of magnitude, and indicates that the CNO process was certainly important in the thermonuclear runaway event.

A more detailed analysis of all three novae, for which in part simultaneous optical spectroscopy and high resolution IUE spectra have been obtained, is in progress and will be published elsewhere.

\section{Acknowledgements}

Special thanks are due to Drs G. Shaviv and A. Cassatella for helpful comments. We appreciate the competent support of the staff of the ESA-Vilspa IUE ground station in Villafranca. This research was partly supported by the Deutsche Forschungsgemeinschaft grants Ra 136/10-2 and Ra 136/11-1.

\section{References}

Andersen, P. H., Borra, E. F., and Dubas, O. V.: 1971, Publ. Astron. Soc. Pacific 83, 5.

Blades, J. C. and Drechsel, H.: 1982, IAU Circ., No. 3741.

Cassatella, A.: 1983, private communication.

Drechsel, H., Rahe, J., Wargau, W., Blades, J. C., Cacciari, C., and Wamsteker, W.: 1982, IAU Circ., No. 3736.

Honda, M.: 1982, IAU Circ., No. 3733.

Liller, W.: 1983, IAU Circ., No. 3764.

Seaton, M. J.: 1979, Monthly Notices Roy. Astron. Soc. 187, 73.

Snijders, M. A. J., Seaton, M. J., and Blades, J. C.: 1982, in Proc. Third European IUE Conference, Madrid, ESA SP-176, p. 177

Stickland, D. J., Penn. C. J., Seaton, M. J., Snijders, M. A. J., Storey, P. J., and Kitchin, C. R.: 1979, in Proc. of The First Year of IUE, Londen, p. 63.

Stickland, D. J., Penn, C. J., Seaton, M. J., Snijders, M. A. J., and Storey, P. J. : 1981, Monthly Notices Roy. Astron. Soc. 197, 107. 